PROCEEDINGS OF THE

AMERICAN MATHEMATICAL SOCIETY

Volume 88, Number 2, June 1983

\title{
EQUIVARIANT COVERING SPACES AND COHOMOLOGY
}

\author{
STEFAN WANER
}

\begin{abstract}
The Bredon cohomology of classifying spaces for categories of equivariant covering spaces is considered and shown to correspond to derived functors for the coefficient systems of the Bredon theory.
\end{abstract}

Introduction. If $A$ is a finite group, then one has the classical result that $B A$, the classifying space for principal $A$-bundles, has cohomology equal to the cohomu. 'gy of $A$. The purpose of this note is to detect the algebraic invariant which measures the $G$-equivariant (Bredon) cohomology of equivariant classifying spaces for $G$ a finite group.

Specifically, we consider important categories of $G$-covering spaces, formulate universal classifying spaces for such objects, and consider their Bredon cohomology. We then realize this cohomology as an algebraic invariant which may be computed via explicit Bar resolutions (Theorem 3.4). These invariants turn out to be derived (Ext) functors associated with the coefficient system of the cohomology.

The author would like to thank the referee for his thorough reading of the original manuscript (which contained erroneous claims) and for his suggestions, incorporated here.

1. Equivariant covering spaces. Let $A$ be a finite group. By a $(G, A)$-covering space $p: E \rightarrow B$, we shall mean a covering space with structure group $A$ such that $p$ is equivariant with respect to given $G$-actions on $E$ and $B$. We also require that, if $b \in B$, then the action of $G_{b}=\{g \in G: g b=b\}$ on $p^{-1}(b)$ is given via some homomorphism $G_{b} \rightarrow A$.

An important way in which such covering spaces arise is given as follows. Let $X$ be a completely regular left $G$-space with a right $A$-action such that the $A$-action is free and commutes with the $G$-action (so that $(g x) a=g(x a)$ for each $g \in G, x \in X$ and $a \in A$ ). The orbit space $X / A$ is then automatically a $G$-space, with the projection $p: X \rightarrow X / A G$-equivariant. Denote $p(x)$ by $[x]$. Then, for each $x \in X$, $G_{[x]}$ acts on $p^{-1}([x]) \cong A$. Such an identification $\eta: A \rightarrow p^{-1}([x])$ may be obtained by restricting a coordinate function, and two such differ by a translation (that is, if $\xi$ is another, then $\left(\xi^{-1} \circ \eta\right)(a)=a_{0} a$ for some fixed $\left.a_{0}\right)$.

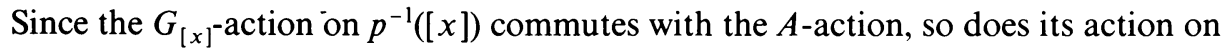
$A$ (via $\eta$ ). Thus, for each $h \in G_{[x]}, h a=\left(h e_{A}\right) a$, where $e_{A}$ is the identity in $A$. The

Received by the editors December 3, 1981 and, in revised form, August 28, 1982.

1980 Mathematics Subject Classification. Primary 54H15.

Key words and phrases. Equivariant covering spaces, bar resolution, Bredon cohomology, coefficient system, Hecke functor. 
assignment $h \rightarrow h e_{A}$ is then a homomorphism $\omega: G_{[x]} \rightarrow A$. Indeed, if $h_{1}$ and $h_{2}$ are in $G_{[x]}$, then $\omega\left(h_{1} h_{2}\right)=\left(h_{1} h_{2}\right) e_{A}=h_{1}\left(h_{2} e_{A}\right)=h_{1}\left(e_{A} h_{2} e_{A}\right)=\left(h_{1} e_{A}\right)\left(h_{2} e_{A}\right)$ since the $G_{[x]^{-}}$and $A$-actions commute. One therefore has, for each $h \in G_{[x]}, h a=\omega(h) a$ for some homomorphism $\omega: G_{[x]} \rightarrow A$. Since $\omega$ depends on the choice of the coordinate function $\eta$, denote $\omega$ by $\omega_{\eta}$. If $\xi$ is another coordinate function, then $\xi^{-1} \eta\left(e_{A}\right)=a_{0}$, and the action on $A$ determined by $\xi$ is given by $h *_{\xi} e_{A}=$ $a_{0}\left(h *_{\eta} e_{A}\right) a_{0}^{-1}$, where $*_{\xi}$ and $*_{\eta}$ denote the two actions in question. Thus $\omega_{\xi}(h)=$ $a_{0} \omega_{\eta}(h) a_{0}^{-1}$, and the two actions are conjugate.

The projection $p: X \rightarrow X / A$ is thus an example of a $(G, A)$-covering space. This example is, in a sense, the most general example; if $F$ is a left $A$-space, then the projection $p: X \times{ }_{A} F \rightarrow X / A$ may be seen to be a $(G, A)$-covering space, and all $(G, A)$-covering spaces arise in this way, just as in the nonequivariant case.

If the fixed-sets of the base $X / A$ are connected, then one may select a $G$-fixed point $\left[x_{0}\right]$ and a fixed identification $\eta_{0}: A \rightarrow p^{-1}\left(\left[x_{0}\right]\right)$, and use the lifting property to obtain fixed identifications $A \rightarrow p^{-1}([x])$ for all fibers. One is therefore given a fixed homomorphism $\sigma: G \rightarrow A$, such that $G_{[x]}$ acts on $p^{-1}([x])$ via $\sigma \mid G_{[x]}$ for each $x \in X$. The situation is the same for arbitrary $(G, A)$-covering spaces $p: E \rightarrow B$ with each $B^{H}$ connected.

If the fixed-sets of $B$ are disconnected, the action of some isotropy subgroup on two distinct fibers may be unrelated in general. Further, the homomorphisms $G_{b} \rightarrow A$ need not extend to a $G$-action. As a consequence, the equivariant classifying space for such objects is complicated, involving as it must all possible actions by subgroups of $G$ on the fiber. We shall therefore restrict attention to the following (simpler) kind of $(G, A)$-covering space.

Definition 1.1. Let $F$ be a finite discrete space with $A \subset \operatorname{Aut}(F)$, and let $\sigma$ : $G \rightarrow A$ be a fixed homomorphism. Then a $(G, A ; \sigma)$-covering space $p: E \rightarrow B$ is a $(G, A)$-covering space with fiber $F$ such that, for each $b \in B, p^{-1}(b)$ and $F$ are $G_{b}$-homomorphic, where $G$ acts on $F$ via $\sigma$.

Of course, this definition is meaningful whether or not $B$ has connected fixed-sets.

In [W4] we describe an analogous concept for $G$-vector bundles, and construct classifying spaces for such bundles. Mutatis mutandi, one has the following descriptions of the classifying space for $(G, A ; \sigma)$-coverings.

Proposition 1.2. Let $G$ act on $A$ from the left by the action $g \cdot a=\sigma(g) a \sigma(g)^{-1}$ for $a \in A$. Then $B A$, with the $G$-action inherited from the action on $A$, is the classifying space for $(G, A ; \sigma)$-coverings.

Further, if one uses, as one's model for $B A$, the two-sided geometric Bar construction of $[\mathbf{M 1}], B A=B(*, A, *)$, then the universal $(G, A ; \sigma)$-covering with fiber $F$ over $B A$ is given by the natural projection $p: B(*, A, F) \rightarrow B(*, A, *)$. Here, $G$ acts on $F$ via $\sigma$ and on $A$ as above.

Let $G$ and $A$ act, respectively, from the left and right on $E A=B(*, A, A)$ via the action

$$
(g, a) \cdot\left(*\left[a_{n}, \ldots, a_{1}\right] a_{0}\right)=*\left[\sigma(g) a_{n} \sigma(g)^{-1}, \ldots, \sigma(g) a_{1} \sigma(g)^{-1}\right] \sigma(g) a_{0} a,
$$

for $g \in G$ and $a \in A$, on the simplicial level. 
Then the actions of $G$ and $A$ are compatible, so that one has a $(G, A)$-biaction, or equivalently, a $(G \times \bar{A})$-action, where $\bar{A}=A^{\text {opposite }}$. The projection

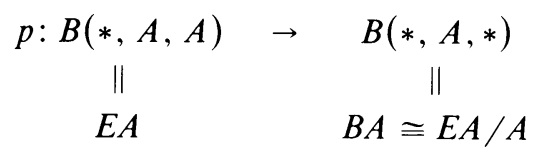

is a $(G, A ; \sigma)$-covering, to be thought of as the universal $(G, A ; \sigma)$-principal covering.

In order to keep track of $G$ and $\sigma$, we shall sometimes denote $E A$ by $E_{G}^{\sigma} A$ and $B A$ by $B_{G}^{\sigma} A$.

As examples, $B_{G}^{l} G$ is $B G$ acted upon by $G$ via conjugation, while if $G$ has order prime to $p$, then $B_{G}^{\sigma} Z_{p}$ must have $\sigma$ trivial and hence a trivial $G$-action.

We would like to compute the Bredon equivariant cohomology of $B_{G}^{\sigma} A$, with coefficients in suitable Bredon coefficient systems. To do this, we shall need information about the orbit structure of the $(G \times \bar{A})$-space $E_{G}^{\sigma} A$.

If $\psi: G \rightarrow A$ is a homomorphism and $H \subset G$, let $H_{\psi} \subset G \times \bar{A}$ be the subgroup $\left\{\left(h, \psi(h)^{-1}\right)\right\}_{h \in H}$.

Proposition 1.3. Denote by $\psi_{a}$ the homomorphism $G \rightarrow A$ given by $g \rightarrow a^{-1} \sigma(g) a$, for $a \in A$. Then any point $x$ in $E_{G}^{\sigma} A$ has isotropy subgroup of the form $H_{\psi_{a}}$ with $H \subset G$ the isotropy subgroup of $p(x)$.

Proof. Since the action on simplicial coordinates is trivial, it suffices to compute isotropy on the simplicial space level where it is obvious.

Returning to the case of the $(G, A)$-covering space $p: X \rightarrow X / A$, one sees again that for $x \in X, \quad(G \times \bar{A})_{x}=\left\{\left(h, a_{1}^{-1} \sigma(h) a_{1}\right): H \in G_{[x]}\right\}$, (where $\eta\left(a_{1}\right)=x$ ) $=\left(G_{[x]}\right)_{\psi_{a}}$.

We shall concern ourselves with the computation of Bredon cohomology $H_{G}^{*}(X / A ; T)$ for suitable coefficient systems $T$.

2. Hecke functors and equivariant cohomology. If $\mathscr{F}$ is a family of subgroups of $G$ closed under conjugation, let $\mathbf{Z}(G, \mathcal{F})$ denote the category whose objects are the $G$-sets $G / H$ with $H \in \mathcal{F}$ and whose morphisms are the $\mathbf{Z} G$-module morphisms $\mathbf{Z} G / H \rightarrow \mathbf{Z} G / K$, where $\mathbf{Z} G / J$ denotes the free module on the $G$-set $G / J$.

Now let $\mathcal{F}^{\prime}$ be a family, closed under conjugation, of subgroups of $G \times \bar{A}$ of the form $H_{\psi}$ for $H \subset G$. Define an associated family $\mathscr{F}$ of subgroups of $G$ by taking $\mathcal{F}=\left\{\pi(Q): Q \in \mathcal{F}^{\prime}\right.$ and $\pi: G \times \bar{A} \rightarrow G$ the projection $\}$. As an example, one might take $\mathscr{F}^{\prime}=\left\{H_{\psi_{a}}: H \subset G\right.$ and $\left.a \in A\right\}$. If $\mathscr{F}^{\prime}$ is closed under passage to subgroups, then so is $\mathcal{F}$, and one has a projection. $P: \mathbf{Z}\left(G \times \bar{A} ; \mathcal{F}^{\prime}\right) \rightarrow \mathbf{Z}(G ; \mathcal{F})$ which assigns to $(G \times \bar{A}) / Q$ the object $G / \pi(Q)$ and to $f: \mathbf{Z}(G \times \bar{A}) / Q \rightarrow \mathbf{Z}(G \times \bar{A}) / N$ the composite

$$
\mathbf{Z} G / \pi(Q) \stackrel{\iota}{\rightarrow} \mathbf{Z}(G \times \bar{A}) / Q \stackrel{f}{\rightarrow} \mathbf{Z}(G \times \bar{A}) / N \stackrel{\nu}{\rightarrow} \mathbf{Z} G / \pi(N)
$$

where $\iota(g \pi(Q))=\left(g, \psi(g)^{-1}\right) Q$ for any $\psi$ such that $Q$ has the form $H_{\psi}$, and where $\nu$ is given by $(g, a) N \rightarrow g \pi(N)$. That this composite is independent of the choice of $\psi$ and that $P$ respects composition is easy to check. 
As in [W1], there are associated notions of Hecke functors.

Definition 2.1. $A(G, \mathscr{F})$-Hecke functor is an additive contravariant functor $T$ : $\mathbf{Z}(G, \mathcal{F}) \rightarrow \mathbb{Q} b$, the category of abelian groups. Similarly, one has the notion of a $\mathbf{Z}\left(G \times \bar{A} ; \mathscr{F}^{\prime}\right)$ Hecke functor.

The collection of $(G, \mathscr{F})$-functors forms a category $\mathscr{O}(G, \mathscr{F})$, where the morphisms are taken to be the natural transformations.

Notice that $P$ induces a functor $P^{*}: \mathfrak{T}(G ; \mathscr{F}) \rightarrow \mathscr{T}\left(G \times \bar{A} ; \mathscr{F}^{\prime}\right)$ in a natural way.

EXAMPLES 2.2. (i) If $\hat{R}$ is a $\mathbf{Z} G$-module, one has an associated $(G, \mathscr{F})$-Hecke functor $\hat{R}$ given by $\hat{R}(G / H)=\hat{R}^{H} \cong \operatorname{Hom}_{\mathbf{Z} G}(\mathbf{Z} G / H, R)$ for $H \in \mathscr{F}$. If $f: \mathbf{Z} G / K \rightarrow$ $\mathbf{Z} G / H$ is in $\mathbf{Z}(G ; \mathscr{F})$, then one defines $\hat{R}(f)$ to be composition with $f$. In particular, when $R=\mathbf{Z}$ with the trivial $G$-action, then $\hat{\mathbf{Z}}(G / H)=\mathbf{Z}$ for each $H \in \mathscr{F}$.

(ii) If $R$ is a $\mathbf{Z}(G \times \bar{A})$-module, then the construction in (i), with $G$ replaced by $G \times \bar{A}$, gives an associated $\left(G \times \bar{A}, \mathscr{F}^{\prime}\right)$-Hecke functor.

Now let $M_{*}$ be a d.g. $(G \times \bar{A}, \mathscr{F})$-Hecke functor, and define its cohomology, $H^{*}\left(M_{*} ; T\right)$, with coefficients in $T \in \mathscr{N}(G \times \bar{A} ; \mathscr{F})$, to be the cohomology of the Hom sets $\Re\left(G \times \bar{A} ; \mathscr{F}^{\prime}\right)\left(\Re_{*}, T\right)$.

EXAMPLES 2.3. (i) If $X$ is a $(G \times \bar{A})$-CW complex with orbit-types $(G \times \bar{A}) / Q$ for $Q \in \mathcal{F}^{\prime}$, then the chain complex $C_{*}(X)$ of $X$ is a $\mathbf{Z}(G \times \bar{A})$-module, and hence, by Example 2.2(ii), a $\left(G \times \bar{A}, \mathscr{F}^{\prime}\right)$-Hecke functor. Define $\mathscr{N} H_{G \times \bar{A}}^{*}(X ; T)_{\mathscr{F ^ { \prime }}}$ to be $H^{*}\left(C_{*}(X) ; T\right)$ for $T \in \mathscr{T}\left(G \times \bar{A} ; \mathscr{F}^{\prime}\right)$. If $\mathscr{F}^{\prime}$ is closed under passage to subgroups, we shall see that this coincides with Bredon cohomology, provided $T$, regarded as a $(G \times \bar{A})$-equivariant coefficient system (see [B1]), extends to one defined on $(G \times \bar{A}) / K$ for all $K \subset G \times \bar{A}$. More importantly, we shall see in $\S 3$ that

$$
\text { } \Re H_{(G \times \bar{A})}^{*}\left(X ; P^{*} T\right) \cong H_{G}^{*}(X / A ; T)
$$

for a Hecke functor $T$.

(ii) Let $T \in \mathscr{R}(G \times \bar{A} ; \mathscr{F})$ be such that each $T(G / H)$ is free. One then has a projective resolution of $T$ in $\mathscr{T}(G \times \bar{A} ; \mathcal{F})$ given explicitly by the two-sided algebraic Bar construction in [W1] with $G$ replaced by $G \times \bar{A}$. If we denote this resolution by $W_{*}(T, \mathscr{F})$, then set $H_{(G \times \bar{A})}^{*}\left(T ; T^{\prime}\right)_{\mathscr{F}}=H^{*}\left(W_{*}(T ; \mathscr{F}) ; T^{\prime}\right)$ for $T^{\prime} \in$ $\mathfrak{R}\left(G \times \bar{A} ; \bar{F}^{\prime}\right)$ is clearly the derived functor Ext* in the category $\mathfrak{N}\left(G \times \bar{A} ; \bar{F}^{\prime}\right)$ and generalized the notion of the cohomology of $G$. Indeed, if $A=\{e\}$ and $\mathscr{F}$ is the collection of all subgroups of $G \times \bar{A} \cong G$, then $H_{(G \times \bar{A})}^{*}(\hat{\mathbf{Z}} ; \hat{R})_{\mathscr{F}} \cong H^{*}(G ; R)$.

REMARK 2.4. The requirement that $T$ be a Hecke functor may be dropped. In [W4], we deal with arbitrary coefficient systems $T$, and this involves a formal change of category from $\mathbf{Z}(G ; \mathcal{F})$. The restriction here to Hecke functors demonstrates the direct generalization of $H^{*}(G ; R)$.

3. Relationship with Bredon cohomology. As promised in $\S 2$, we consider the theory $H_{(G \times \bar{A})}^{*}(X ; T)_{\mathscr{F}}$, for $\mathcal{F}^{\prime}$ as in $\S 2$.

By $[\mathbf{W} 1,3.1]$ one has

Proposition 3.1. Let $X$ be any $(G \times \bar{A})$-CW complex with cells of type $(G \times \bar{A}) / Q$ with $Q \in \mathcal{F}^{\prime}$. Then $\mathfrak{T}_{(G \times \bar{A})}^{*}(X ; T)_{\mathscr{F}} \cong H_{(G \times \bar{A})}^{*}(X ; T)$, Bredon cohomology of $X$ with coefficients in the coefficient system $T$, where we take $T((G \times \bar{A}) / Q)=0$ if $Q \notin \mathcal{F}^{\prime}$. 
Corollary 3.2. Let $X$ be as in 3.1. Then $\mathfrak{N} H_{(G \times \bar{A})}^{*}\left(X ; P^{*} T\right)_{\mathscr{F}^{\prime}} \cong H_{G}^{*}(X / A ; T)$, where we regard $T$ as the Bredon coefficient system as in Example 2.3(i).

Proof. By the proposition, we already have

$$
\Re H_{(G \times \bar{A})}^{*}\left(X ; P^{*} T\right)_{\mathscr{F}^{\prime}} \cong H_{(G \times \bar{A})}^{*}\left(X ; P^{*} T\right)
$$

and we are left with having to show that $H_{(G \times \bar{A})}^{*}\left(X ; P^{*} T\right) \cong H_{G}^{*}(X / A ; T)$, and this is standard. Let $\pi: G \times \bar{A} \rightarrow G$ be projection. Then $p: X \rightarrow X / A$ is a $\pi$-map, and, we claim, gives rise to an induced homomorphism in equivariant cohomology. Indeed, the identity maps 1: $T(G / \pi(Q)) \rightarrow P^{*} T((G \times \bar{A}) / Q)$ form a natural transformation with respect to $\pi$ (see [I1, p. 45]), and so, by Corollary II, 1.5 in [I1], we have induced homomorphisms

$$
(P, \pi, 1)^{*}: H_{G}^{n}(X / A ; T) \rightarrow H_{(G \times \bar{A})}^{n}\left(X ; P^{*} T\right)
$$

for all $n$. When $X=(G \times \bar{A}) / Q$, the projection $p: X \rightarrow X / A$ is the natural map $p_{\pi}$ : $(G \times \bar{A}) / Q \rightarrow G / \pi(Q)$, and by [I1, II, 1.6], $(p, \pi, 1)^{*}$ corresponds for such an $X$, to the identity map 1: $T(G / \pi(Q)) \rightarrow P^{*} T((G \times \bar{A}) / Q) \stackrel{\text { def }}{=} T(G / \pi(Q))$ (under the dimension axiom isomorphism). It now follows that $(p, \pi, 1)^{*}$ is an isomorphism for any $(G \times \bar{A})$-CW complex $X$.

Corollary 3.3. Let $X$ be as in Proposition 3.1, with $X / Q$ Z-acyclic for each $H \in \mathscr{F}^{\prime}$. Then

$$
H_{G}^{*}(X / A ; T) \cong H_{(G \times \bar{A})}^{*}\left(\hat{\mathbf{Z}} ; P^{*} T\right)_{\mathscr{F}{ }^{\prime}},
$$

provided $\mathcal{F}^{\prime}$ is closed under passage to subgroups. Here, $\hat{\mathbf{Z}}$ denotes the constant functor in $\mathscr{T}\left(G \times \bar{A} ; \mathscr{F}^{\prime}\right)$.

Proof. Since $C_{*}\left(X / H_{\psi}\right) \cong C_{*}(X)^{H_{\psi}}$ is a resolution of $\mathbf{Z}$ for each $H \in \mathcal{F}^{\prime}$ it follows that, in $\Re\left(G \times \bar{A} ; \mathscr{F}^{\prime}\right), C_{*}(X)$ is a projective resolution of $\mathbf{Z}$. Indeed, one notes that the assignment $C_{*}(X /-) \rightarrow H_{0}(X /-; \mathbf{Z})$ is in $\Re\left(G \times \bar{A} ; \mathscr{F}_{\psi}\right)$, the latter functor belonging there by virtue of the isomorphisms $H_{0}\left(X / H_{\psi} ; \mathbf{Z}\right)=$ $H_{0}\left(C^{*}(X)^{H_{\psi}} ; \mathbf{Z}\right)$. The result now follows by the definition in Example 2.3 (ii).

We may now conclude the main result. Let $\sigma: G \rightarrow A$ be a homomorphism, take $\psi$ to be the collection $\left\{\psi_{a}: a \in A\right\}$ associated with $\sigma$, as in Proposition 1.3, and take $\mathscr{F}$ to be the collection of all subgroups of $G$, and $\mathscr{F}^{\prime}=\mathscr{F}_{\psi}$.

THEOREM 3.4. With the above conventions,

$$
H_{G}^{*}\left(B_{G}^{\sigma} A ; T\right) \cong H_{(G \times \bar{A})}^{*}\left(\hat{\mathbf{Z}} ; P^{*} T\right)_{\mathscr{F}_{\psi}} .
$$

Proof. By Corollary 3.3, it suffices to show that $E_{G} A / H_{\psi_{a}}$ is contractible for each $H \subset G$ and $a \in A$. But $\left(E_{G}^{\sigma} A\right)^{H_{\psi_{a}}}=B(*, A, A)^{H_{\psi_{a}}}=B\left(*, C_{A}(H), C_{A}(H) a\right)$, where $C_{A}(H)=\left\{a \in A: \sigma(h) a \sigma(h)^{-1}=a\right.$ for each $\left.h \in H\right\}$, and $C_{A}(H) a$ denotes the right coset of $a$ in $A / C_{A}(H)$. Since $B\left(*, C_{A}(H), C_{A}(H) a\right)$ is homeomorphic to the contractible space $E C_{A}(H)$, it follows that $\left(E_{G}^{\sigma} A\right)^{H_{\psi_{a}}}$ is contractible for each $H$ and $a$, whence, by the $H_{\psi_{a}}$-equivariant version of Whitehead's theorem, $E_{G}^{\sigma} A$ is $H_{\psi_{a}}$-equivariantly contractible, so that $E_{G} A / H_{\psi_{a}}$ is contractible.

REMARKS 3.5. (i) If $\mathscr{F}$ is not closed into passage to subgroups, the argument in the proof of 3.4 fails as the Whitehead theorem does not apply. 
(ii) If $G=\{1\}$, then the theorem implies the well-known result $H^{*}(B A ; R) \cong$ $H^{*}(A ; R)$ for any trivial $A$-module $R$.

(iii) The theorem implies that $H_{G}^{*}\left(B_{G}^{\sigma} A ; T\right)$ is computable from a purely algebraic viewpoint via the use of a two-sided Bar construction.

\section{BIBLIOGRAPHY}

[B1] G. E. Bredon, Equivariant cohomology theories, Lecture Notes in Math. vol. 34, Springer-Verlag, Berlin, 1967.

[I1] S. Illman, Equivariant singular homology and cohomology. I, Mem. Amer. Math. Soc. No. 156 (1975), 1-74.

[M1] J. P. May, Classifying spaces and fibrations, Mem. Amer. Math. Soc. No. 155 (1975).

[W1] S. Waner, A generalization of the cohomology of groups, Proc. Amer. Math. Soc. 85 (1982), 469-474.

[W2] __ Unoriented equivariant $R O(G)$-graded bordism, Univ. of Virginia, 1981, preprint.

[W3] _ Oriented equivariant spherical fibrations, Trans. Amer. Math. Soc. 271 (1982), 313-324.

[W4] Three topological categories of G-sets, Univ. of Virginia, 1981, preprint.

Department of Mathematics, Hofstra University, Hempstead, New York 11550 